\title{
Selecting appropriate dynamic model for elastomeric engine mounts to approximate experimental FRF data of them
}

\author{
K. Jahani ${ }^{1, a}$ \\ ${ }^{1}$ Faculty of Mechanical Engineering, University of Tabriz, 29 Bahaman blvd., Tabriz, Iran
}

\begin{abstract}
In this paper, the capabilities of different dynamic analytical models to approximate experimentally measured FRFs of elastomeric engine mounts of a passenger car are investigated. Artificial neural networks is used in identifying the dynamic characteristics of each model. Impact hammer test is implemented to extract measured FRFs and harmonic analysis is used to get the counterpart response of the models. Here linear and orthotropic material properties are considered for elastomeric media. The frequency response functions of updated models are compared with experimentally detected ones and advantages and limitations of each model to simulate the real dynamic behaviour of elastomeric engine mounts are discussed
\end{abstract}

\section{Introduction}

Elastomeric engine mounts are still implemented in vehicles because of being compact, costeffective and maintenance free. Bonded elastomeric mounts are known to provide more consistent performance and longer life [1]. One of the major functions of engine mounts is to isolate the unbalanced engine disturbance force from the vehicle structure.

Predicting the dynamic characteristics of engine mounts by experiments is necessary to analysis the NVH performance of a vehicle by CAE tools in the early stage of a new vehicle design. Usually two types of experiments may be conducted to predict the dynamic characteristic of elastomeric like materials. The first types are tensile tests which are static or low frequency tests. The second types are resonant tests which can predict wide frequency range characteristics of the material [2].

The behaviour of the elastomers is very complex. Considering nonlinearities of hyperelastic behaviours of elastomers and also anisotropic material properties lead to difficulties in performing harmonic analysis of FE model. On macroscopic scale, elastomers usually behave elastically isotropic initially, and anisotropic at finite strain. Under essentially monotonically loading condition, however, a larger class of the elastomers can be approximated by an isotropic assumption [3].The nonlinearity problem can be overcome when the behaviour of the joint (here the elastomeric material of engine mount) can be assumed linear in the neighbourhood of a given operating condition [4].

To tackle the above mentioned difficulties, in present studies it is assumed that elastomeric material has orthotropic and linear property in small deformation condition. Engine mounts in fact are joints which attach engine block to body frame of a vehicle. Usually Kelvin-Voight model (or

a e-mail : ka_jahani@tabrizu.ac.ir

This is an Open Access article distributed under the terms of the Creative Commons Attribution-Noncommercial License 3.0, which permits unrestricted use, distribution, and reproduction in any noncommercial medium, provided the original work is properly cited. 
different combinations of springs and dashpots), beam model and 3D finite element model are used to analyses joint structures [5].In this paper, the capabilities of these models to predict the dynamic behaviour of elastomeric engine mounts are investigated and compared.

\section{Methodology}

The frequency response function of an elastomeric engine mount in free-free condition is approximated by different dynamic models. The investigated engine mount is presented in figure 1 . The engine mount is composed of the rubber (middle part which will be called 'elastomeric media' within the text), plates at bottom an top, a bracket at the bottom and a bolt on the top.

The measured static stiffness of the mount in three orthogonal directions (see figure 2) are presented in Table 1 . These values will be used to statically update the parameters of the investigated models in elastomeric media.

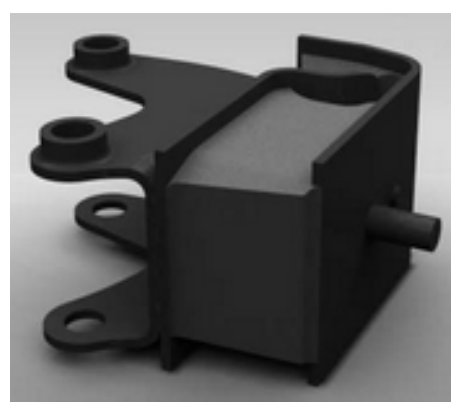

Fig. 1 the investigated engine mount

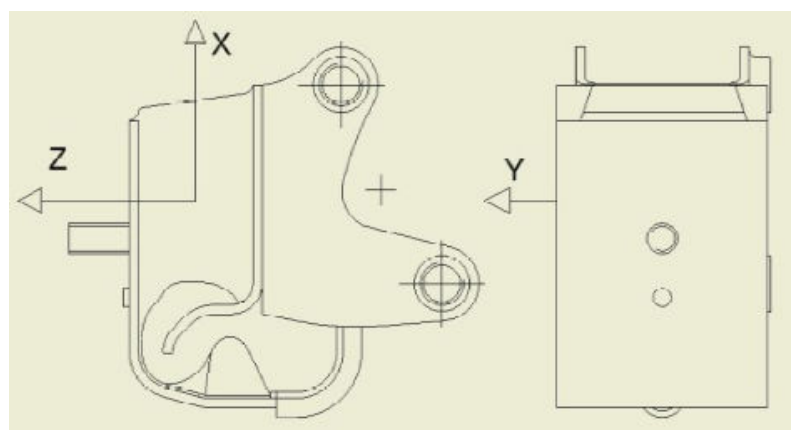

Fig. 2 static stiffness measuring directions

Table 1 Measured linear static stiffness in 3 directions

Measured linear static stiffness [N/mm]

\begin{tabular}{|l|l|l|}
\hline $\mathrm{X}$ & $\mathrm{Y}$ & $\mathrm{Z}$ \\
\hline 71 & 161 & 462 \\
\hline
\end{tabular}




\subsection{Different Analytical (FE) models of the elastomeric media of the engine mount}

Three different categories of models for elastomeric part of the engine mount are studied in this paper which includes: spring-dashpot model, 3-D elastic beam model and elastic solid model.

\subsubsection{Spring-dashpot model}

Common types of spring- dashpot models which are used in modelling of engine mounts are Kelvin-Voight, Zener and Zener models .considering these three models of spring-dashpot, the Zener model leads to the best fit of the frequency -dependent behaviour of the rubber mounts [6,7]. To avoid of more complexity, the Zener model is chosen for investigation in this study.

The elastomeric media of the investigated engine mount is modelled by 8 Zener model such that each side of elastomeric surface has two oblique Zener elements. The constructed FE model is presented in figure 3. The stiffnesses obtained from static tests in three directions are used for initially updating the model. Tow sets values are obtained for parameter, $\mathrm{k}$. for 4 side elements this value is: $40000 \mathrm{~N} / \mathrm{m}$ and for the other 4 front and back elements the value is: $990000 \mathrm{~N} / \mathrm{mi}$. identified stiffnesses after updating are presented in Table 2.

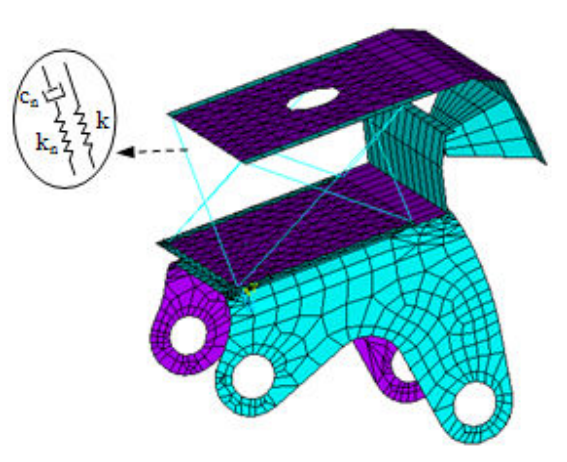

Fig. 3. Elastomeric media replaced by 8 Zener elements

Table 2 identified linear static stiffness in 3 directions

Replacing elastomeric media by Zener elements

Measured linear static stiffness [N/mm]

\begin{tabular}{|c|c|c|}
\hline $\mathrm{X}$ & $\mathrm{Y}$ & $\mathrm{Z}$ \\
\hline 86 & 94 & 457 \\
\hline \multicolumn{3}{|c|}{ Difference with test (\%) } \\
\hline 21 & -42 & -1 \\
\hline
\end{tabular}

\subsubsection{Beam model with damping}

In the present model, the elastomeric media of the engine mount is modelled as a continuous elastic 3-D beam with density $\rho$ and structural damping characterized by the loss factor $\eta$. More than one beam element is needed to make correct model of an engine mount. Here, 5 beam elements are used 
in constructing of FE model of the investigated engine mount. The FE model of engine mount is presented in figure 4.

3-D beam elements have elasticity in three directions, so these elements may be able to simulate the dynamic behaviour of the elastomeric media (rubber portion of the engine mount) under the applied loads. The implemented beam elements have isotropic material properties; therefore, the available parameters to initially update the model with static stiffness tests are Young's modulus and section parameters of beams. The stiffnesses of the engine mounting in three directions after updating are presented in Table 3. Since the beam elements don't have the capability of orthotropic material properties so, accurately updating the stiffness of the FE model in three directions is difficult.

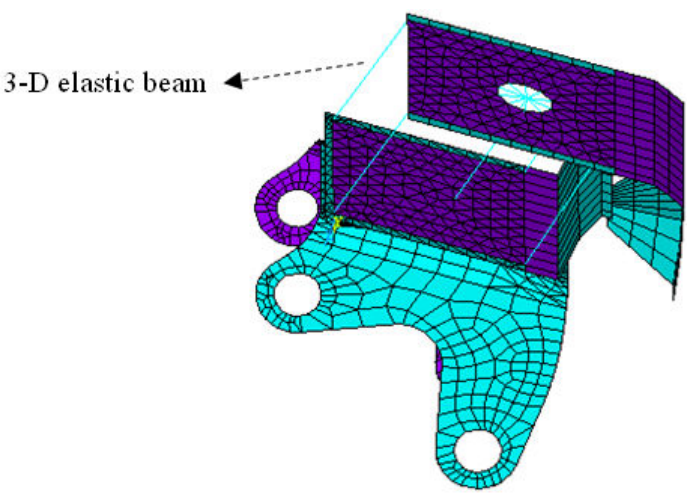

Fig. 4. Elastomeric media replaced by 3-D beam elements

Table 3 identified linear static stiffness in 3 directions using beam elements

\begin{tabular}{|c|c|c|}
\hline \multicolumn{3}{|c|}{$\begin{array}{l}\text { linear static stiffness of the engine mount } \\
\text { after updating using beam elements } \\
\qquad[\mathrm{N} / \mathrm{mm}]\end{array}$} \\
\hline $\mathrm{X}$ & $\mathrm{Y}$ & $\mathrm{Z}$ \\
\hline 104 & 220 & 366 \\
\hline \multicolumn{3}{|c|}{ Difference with test (\%) } \\
\hline 46 & 37 & -21 \\
\hline
\end{tabular}

\subsubsection{Elastic solid model with damping}

Elastic solid elements have the capability to define the orthotropic material model that makes easy to approximate their stiffness characteristics in three directions. Also, they can simulate incompressible behaviour of rubber like materials. Here, 8 node brick elements with the viscoelestic capability are implemented in modelling of engine mount. The FE model of the engine mount is shown in fig. 5.

The static stiffnesses of the engine mount after updating are presented in Table 4. The orthotropic material properties for elastomeric region which lead to identified stiffness were as follow: $\mathrm{EX}=2.7 \mathrm{E}+006[\mathrm{~Pa}] ; \mathrm{EY}=9.8 \mathrm{E}+006[\mathrm{~Pa}] ; \mathrm{EZ}=7 \mathrm{E}+006[\mathrm{~Pa}] ; \mathrm{NUXY}=0.42 ; \mathrm{NUYZ}=0.42 ; \mathrm{NUXZ}=0.42$; $\mathrm{GXY}=9.5 \mathrm{E}+005[\mathrm{~Pa}] ; \mathrm{GYZ}=3.45 \mathrm{E}+006[\mathrm{~Pa}] ; \mathrm{GXZ}=9.5 \mathrm{E}+005[\mathrm{~Pa}]$. 


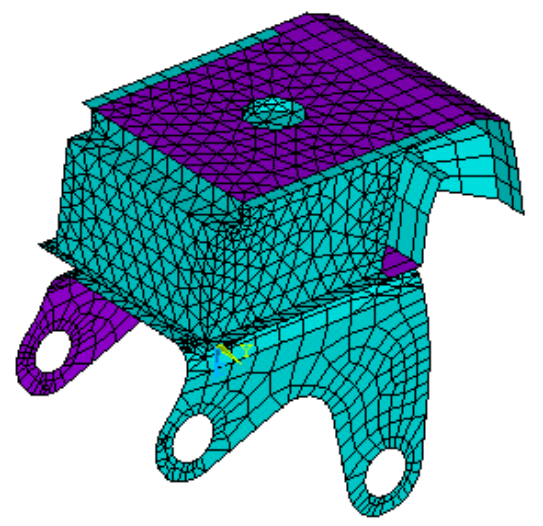

Fig. 5. Elastomeric media modelled by elastic solid elements

Table 4 identified linear static stiffness in 3 directions using solid elements

\begin{tabular}{|}
$\mid \begin{array}{c}\text { linear static stiffness of the engine mount } \\
\text { using beam elements after updating } \\
{[\mathrm{N} / \mathrm{mm}]}\end{array}$ \\
\hline $\mathrm{X}$ & $\mathrm{Y}$ & $\mathrm{Z}$ \\
\hline 77 & 147 & 458 \\
\hline \multicolumn{3}{|c|}{ Difference with test (\%) } \\
\hline 8.5 & -8.7 & -1 \\
\hline
\end{tabular}

\subsection{Measuring the frequency response functions}

Frequency response functions of the investigated engine mount between the bolt and three different points are derived from impact hammer tests. In figure 6, exciting the engine mount in vertical direction (Z-direction) with an impact hammer and measuring the response in the opposite side is presented. Both impulse and response signals are fed into a dual channel analyser and frequency response function are derived from it.

\subsection{Strategy to have better FRFs correlation between the model and dynamic test}

In each model, by changing the damping values of elastomeric media, the frequency response functions of tested points are computed by harmonic analysis. The target is to get more correlations between measured and computed frequencies functions. For instance, in figure 7 , the frequency response functions with different structural damping coefficients for beam element model with exciting the bolt in Y-direction and measuring the response on the lower bracket in the same direction is illustrated. As it can be seen from this figure, the FRF with the structural damping coefficient value 0.00005 has more correlation with the counterpart achieved from the modal test. Radial bases function neural networks is implemented to fined the best value of damping. However, there is still need to improve the performance of the model(s) especially in low frequencies. 


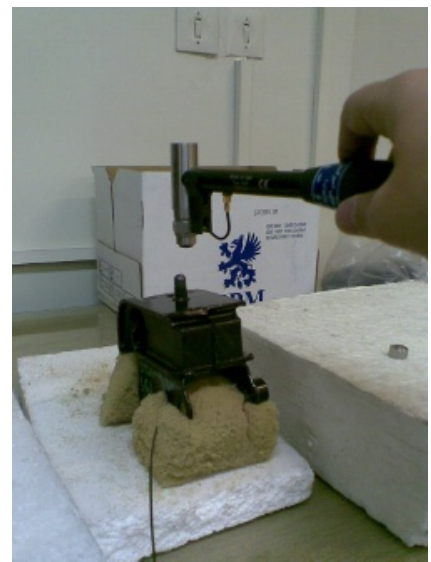

Fig.6. Impact hammer testing in $\mathrm{Z}$ direction

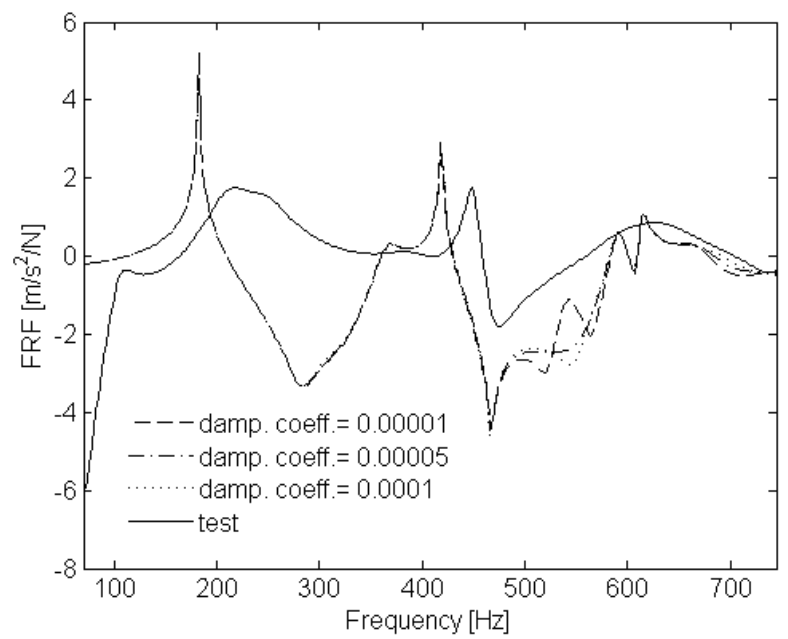

Figure 7 the frequency response functions with different structural damping coefficients for beam element model

\section{3 comparison the performances of the investigated models}

In this section, the capabilities of the above mentioned methods to approximating the experimentally derived FRFs are compared and discussed. For example, the predicted FRFs using the three different models of elastomeric media in Z-direction is shown in the figure 8. The excitation and measuring point locations are the same as illustrated in figure 6 .

Considering the figure 8, it can be concluded that the elastic solid model has the best performance comparing to the other two models and the Spring-Dashpot model is the worst case. Because of making good interconnections within the elastomeric media and also, having the capability of orthotropic material properties, the solid model leads to better showing the internal resonances of the engine mount in free-free testing condition. Considering the Spring-Dashpot model (either Voight or Zener or Generalized Maxwell), may be the free-free testing condition is not suitable to assessing its performance and there is need to conduct forced vibration tests. However, this model still can not simulate the inter connections of the DOFs of the media, so, it is difficult to 
illustrate the internal resonances correctly. The Beam model's performance is between the two others. If one can carefully arrange the Spring -Dashpot or beam elements in different directions with enough interconnections within the elastomeric media may can approve the performance of the model and benefit from having the less DOFs in whole model comparing to the large number of DOFs of Solid model.

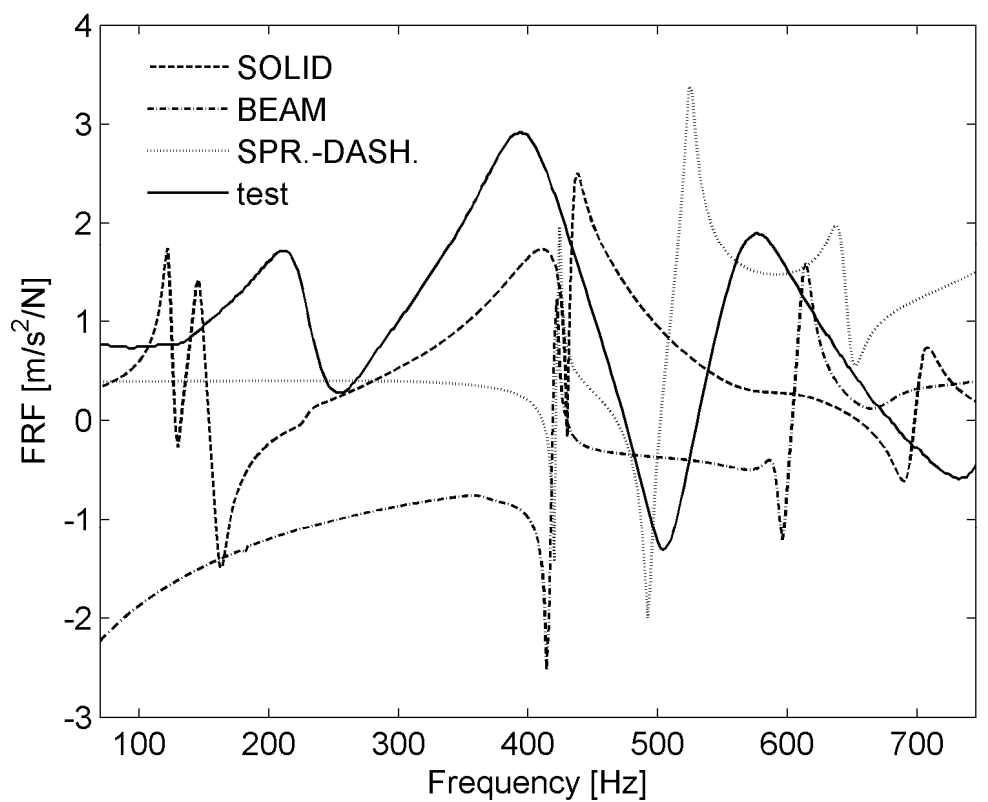

Fig. 8. Comparing the FRFs predicted by the three different models of elastomeric media

\section{4 conclusions}

In this paper, the dynamic capabilities of three different analytical models (elastic solid, beam and spring-dashpot models) of electrometric media of an engine studied. At first, the models statically updated using the measured static stiffnesses at three orthogonal directions. After that, with conducting impact hammer tests in free-free condition, dynamically updating of the models performed. The results showed that the elastic solid model has better performance in predicting the internal resonances of the engine mount comparing to the other two models i.e. Beam and SpringDashpot models. However, still there is need to more work to approve the performances of all of the mentioned models. Weakness and power points of each model in predicting the frequency response functions of the engine mount are discussed.

\section{References}

1. Y. Yu, NG. Naganathan, RV. Dukkipati, Mechanism and Machine Theory J. 36 (2001)

2. K. Jahani, A.S. Nobari, Experimental Mechanics J. 48 (2008)

3. http://www.kxcad.net/ansys/ANSYS/ansyshelp/thy_mat5.html

4. JRF. Arruda, JMC. Santos, SHS. Carneiro IMAC (FL, USA, Fev. 1993)

5. J. Wang, P. Sas ISMA (K.U.L., Leuven, Belgium, 1990)

6. M.J.G. Garcia, N.G. Negrete, J.Vinolas, Int. J. Vehicle Design, 49:4 (2009)

7. Y. Du, R.A. Burdisso, E. Nikolaidis, D. Tiwari, J. Sound and Vibration 268 (2003) 